\title{
Los efectos globo en los cultivos de coca en la Región Andina (1990-2009)*
}

\author{
Balloon effects in coca crops in the \\ Andean region (1990-2009)
}

\section{Os efeitos globo no cultivo de coca na região andina (1990-2009)}

Fecha de recepción: 25 de junio de 2015

Leonardo Raffo López ${ }^{* *}$

Javier Andrés Castro ${ }^{* * *}$

Fecha de aprobación: 9 de diciembre de 2015

Alexander Díaz España ${ }^{* * * *}$

Este artículo es producto de una investigación en la temática del narcotráfico, desarrollada en el contexto de varios proyectos de investigación que contaron con el apoyo de Facultad de Ciencias Sociales y Económicas de la Universidad del Valle y la Vicerrectoría de Investigaciones de esta misma universidad.

* Magíster en Economía, profesor de tiempo completo en el Departamento de Economía de la Universidad del Valle. Cali, Colombia. Dirección postal: Calle 13 No 100-00, Universidad del Valle, Sede Meléndez, edificio 387, oficina 2017. Cali, Colombia. Correo electrónico: leonardo.raffo@correounivalle.edu.co.

.*. Magíster en Economía de la Universidad Nacional de Colombia, profesor de tiempo completo en el Departamento de Economía de la Universidad del Valle. Dirección postal: Calle 13 No 100-00, Universidad del Valle, Sede Meléndez, edificio 387, oficina 2017. Cali, Colombia. Correo electrónico: javier.castro@correounivalle.edu.co.

.... Economista de la Universidad del Valle. Cali, Colombia. Estudiante de último semestre de la Maestría en Economía Aplicada de la Universidad del Valle. Coordinador del "Programa Yumbo Como Vamos", Edificio Centro Empresarial. Dirección postal: Carrera 20 No 33 E bis-94. Yumbo, Colombia. Correo electrónico: alex.ec18@hotmail.com. 


\section{Resumen}

El objetivo de este artículo es poner a prueba estadísticamente la presencia de efectos globo en los cultivos de coca en el territorio geográfico correspondiente a los principales países productores en la región andina (Colombia, Perú y Bolivia) durante el periodo 1990-2009. La metodología empírica se centra en la especificación y estimación de un modelo de ecuaciones simultáneas utilizando el método SUR, el cual permite explicar el comportamiento de las hectáreas cultivadas con coca en función de un conjunto de determinantes. Se concluye que hay evidencia empírica a favor de la presencia de efectos globo en la región andina a lo largo del periodo estudiado.

Palabras clave: efecto globo, ecuaciones estructurales, cultivos ilícitos, modelo SUR, guerra contra las drogas.

Clasificación JEL: K42, C31, N56, Q11.

\section{Abstract}

The aim of this article is to contrast empirically the presence of balloon effects affecting coca crops in the geographic territory corresponding to the main producer countries (Colombia, Perú and Bolivia) of the Andean region during the period 1990-2009. The empirical methodology has been focused in the specification and estimation of a simultaneous equation system using a SUR model. The model attempts to explain the behaviour of the number of hectares cultivated with coca plants as a function of different variables. We conclude that there is empirical evidence supporting the presence of balloon effects in the Andean region during the period of inquiry.

Keywords: balloon effect, structural equations, illicit crops, SUR model, war against drugs.. 


\section{Resumo}

O objetivo deste artigo é testar estatisticamente a presença de efeitos globo sobre os cultivos de coca nos principais países produtores da Região Andina (Colômbia, Peru e Bolívia) durante o período 1990-2009. A metodologia empírica consistiu na estimação de um modelo de equações simultâneas utilizando o método SUR, que pode explicar o comportamento de hectares cultivados de coca, com base em um conjunto de determinantes. Os resultados mostram evidências empíricas a respeito da presença de efeitos globo na região andina durante o período estudado.

Palavras-chave: efeitos globo, modelo SUR, equações estruturais, culturas ilícitas, guerra contra as drogas. 


\section{INTRODUCCIÓN}

Luego de la finalización del Plan Colombia en su primera etapa en el año 2007 se han desarrollado diversos análisis y discusiones sobre su eficacia y los logros alcanzados en la llamada guerra contra las drogas en Colombia y en la región andina (Abadie, Acevedo, Kugler \& Vargas, 2006; Arce \& Reales, 2006; Mejía \& Restrepo, 2008, 2012; Rozo, 2012; Botero, 2013; Rocha, 2011, entre otros). No obstante, no son muchos los trabajos que se han enfocado en el análisis estadístico de la presencia, evolución y consecuencias de los procesos de relocalización geográfica de los cultivos ilegales (de coca y amapola) en esta región como resultado de las políticas de represión en determinadas zonas geográficas; fenómeno que ha sido denominado en la literatura especializada sobre bienes ilegales y narcotráfico como el efecto globo de los cultivos ilegales ${ }^{1}$. Esto resulta paradójico, ya que el análisis de los efectos globo es esencial para entender la evolución de las actividades ilegales en un contexto de represión a su oferta. Es por la presencia de efectos globo que históricamente durante las últimas décadas la disminución de los cultivos en algunas regiones o países ha contrastado con su aumento en otros territorios.

El propósito de este artículo es poner a prueba estadísticamente la presencia de efectos globo en los cultivos con coca en el territorio geográfico correspondiente a los principales países productores de cocaína en la región

\footnotetext{
Algunos trabajos que abordan esta temática son los de Abadie, Acevedo, Kugler y Vargas (2006), Arce y Reales (2006), Bagley (1988, 2012), Moreno-Sánchez, Kraybill y Thompson (2003), Dion y Russler, (2008), Reyes (2014), Dávalos, Bejarano y Correa, (2009), Dávalos et al. (2011), Caicedo (2006), y Rouse y Arce (2006).
} 
andina (Colombia, Perú y Bolivia) durante el periodo 1990-2009, que corresponde al periodo en que se implementó el Plan Colombia en su primera etapa de aplicación, el cual también ha sido denominado como el Plan Colombia I (Rojas, 2007). Por lo tanto, la pregunta de investigación central por responder en lo que sigue es: ¿existe evidencia empírica suficiente para afirmar que el comportamiento de los cultivos con coca en la región se afectó por la presencia de efectos globo? De aquí surgen también otras preguntas específicas: ¿qué factores fueron determinantes en la evolución de los cultivos ilegales entre 1990 y 2009?, ¿incidieron los efectos globo en el nivel de eficacia de las políticas de represión a la oferta de drogas?, ¿cuáles son las características principales y mecanismos de funcionamiento de los efectos globo identificados?

A través de la estimación de un modelo econométrico que explica la evolución de los cultivos con coca para Colombia, Perú y Bolivia, se examina si existe evidencia estadística suficiente para rechazar la hipótesis de que $a$ lo largo del periodo 1990-2009 se han presentado efectos globo en el territorio geográfico correspondiente a los principales países productores de cocaína en la región andina. Para poner a prueba esta hipótesis, se estima de un modelo econométrico de regresiones aparentemente no relacionadas SUR (Seemingly Unrelated Regressions) que explica la evolución de los cultivos con coca en estos países a lo largo del periodo de estudio.

El artículo se ha organizado de la siguiente manera aparte de la introducción. En la segunda sección se explica en qué consiste el efecto globo. En la tercera sección se expone un análisis descriptivo sobre la presencia de efectos globo en los cultivos de coca en Colombia durante el periodo estudiado. En la cuarta se explica la metodología utilizada. En la quinta se presentan los resultados obtenidos. Finalmente se presentan algunas conclusiones.

\section{¿EN QUÉ CONSISTE EL EFEC- TO GLOBO?}

Antes de exponer los hechos que hacen evidente la presencia de efectos globo en la región andina durante la última década del siglo pasado y la primera del siglo XXI, conviene tener presente que en el campo de la economía del crimen y, en particular, en el de la teoría económica de los bienes ilegales, se ha reconocido la notable influencia que tienen los efectos globo en el desarrollo de las diferentes etapas de la cadena productiva de las drogas ilegales en contextos de represión (Nadelmann, 1989; Caulkins, Crawford \& Reuter, 1993; Mejía \& Restrepo, 2013).

El efecto globo se considera como el resultado de la ejecución de políticas de los gobiernos contra la dinámica de 
los mercados de bienes ilegales. Las políticas del Gobierno no logran eliminar las actividades en estos mercados, por el contrario, hacen que haya cambios y respuestas operacionales por parte de los criminales como: el cambio del lugar de la producción, la reducción y descentralización de la producción, y el incremento de la violencia o el amedrentamiento a las instituciones (Arce \& Reales, 2006).

Precisamente, la legislación que busca la prohibición de la producción, la comercialización y el consumo de las drogas ilegales, lleva a los productores a la relocalización de las actividades productivas de estas mercancías y a la producción de otras, para el caso de Colombia (Bagley, 1988). Este autor afirma que el primer indicio de la presencia del efecto globo en Colombia fue observado entre finales de los setenta y principios de los ochenta, como resultado de la militarización y los esfuerzos antidrogas ilegales en la Guajira. Estos provocaron un desplazamiento de los cultivos de droga (marihuana) y las actividades de transporte de las drogas ilegales hacia otras zonas del país como los Llanos orientales y la selva amazónica, más que eliminar estas actividades.

Posteriormente, se encontró evidencia de caídas en los cultivos de coca en Perú y Bolivia e incrementos en las hectáreas de coca en Colombia entre 1987 y 2001 (Moreno-Sánchez,
Kraybill \& Thompson, 2003). Para el periodo 1987-2001 estos autores estiman regresiones econométricas para explicar el área cultivada con coca en Colombia (en hectáreas (ha)) en función de varios factores como el precio de la base de coca en los lugares de cultivo en ese país, el área erradicada en el mismo país, el precio del plátano en los lugares de cultivo (como principal cultivo sustituto de la planta de coca) y el área cultivada con coca en Perú y Bolivia; todas las variables independientes se rezagan un periodo en el tiempo. En el contexto de un modelo de mínimos cuadrados ordinarios (MCO) los autores muestran que la elasticidad de las hectáreas cultivadas con coca en Colombia con respecto a las de Perú y Bolivia no es estadísticamente distinta de uno: un cambio de un $1 \%$ en las hectáreas cultivadas con coca en Perú y Bolivia disminuye en un porcentaje cercano al $1 \%$ las hectáreas cultivadas con coca en Colombia. Esto significa que los esfuerzos por reducir el área cultivada con coca en Colombia se truncan por los aumentos del área cultivada en los otros dos países mencionados, lo que sugiere la presencia de relocalización espacial de los cultivos con pequeños cambios a nivel agregado de la producción (Moreno-Sánchez et al., 2003).

La relocalización de los cultivos de planta de coca también ha sido corroborada a partir de la implementación del Plan Colombia. En el informe Mundial de las Drogas del año 2010 (UNODC, 
2010) se plantea que el reforzamiento de la represión a la oferta de cocaína, a partir del año 2000, redujo las hectáreas de coca cultivadas en Colombia y las desplazó hacia Perú y Bolivia durante esa primera década del siglo XXI (Bagley, 2012). No obstante, según Mejía y Posada (2007), hasta el año 2005 no existía evidencia concluyente que soportara la presencia del efecto globo en los países andinos durante el primer lustro de la década pasada.

Sin embargo, para otros autores (Dion \& Russler, 2008), desde la implementación del Plan Colombia se observa un proceso de dispersión y reconstitución de cultivos en el interior de este país, expandiéndose a zonas que antes de 2005 no contaban con presencia de cultivos de hoja de coca. La presencia de dispersión y descentralización de las actividades ilegales en el interior de un país es otra característica del efecto globo. Para el caso colombiano se demuestra que existen grandes cultivos en áreas más dispersas y alejadas de la presencia de la infraestructura del Estado para escapar de su política de represión y de asistencia (Dion \& Russler, 2008), pero devastando la biodiversidad de bosques tropicales y selvas amazónicas, abundantes e inaccesibles (Dávalos, Bejarano \& Correa, 2009; Dávalos et al., 2011).

Igualmente, trabajos como el de Reyes (2014) dan cuenta de la capacidad de adaptación y la respuesta efectiva de los cultivos de coca en algunos municipios de Colombia al reforzarse la política antidrogas. Esto significa -según el autor-que el incremento de la erradicación de los cultivos lleva a un incremento menos que proporcional en los cultivos de coca, por tanto existe respuesta efectiva pero no inmediata de los cultivadores de hoja de coca. De igual forma, Moreno-Sánchez et al. (2003) encuentran que hasta el año 2001 las áreas cultivadas en los municipios en la mira de las políticas de erradicación, se incrementaron tanto como se intensificaron los esfuerzos de erradicación.

El efecto globo trae externalidades negativas al medio ambiente y la biodiversidad, tal como se mencionó anteriormente. Los trabajos de Dávalos et al. (2009 y 2011) y Rincón-Ruiz y Kallis (2013) dan cuenta de la deforestación por la erradicación y fumigación por aspersión en los cultivos de hoja de coca en las fronteras agrícolas de Colombia. Asimismo, se describen las devastadoras consecuencias sociales de estas políticas de represión a la oferta. La población de los nuevos territorios colonizados es agobiada por incrementos de la violencia, el crimen y el desplazamiento forzado, por ejemplo los indígenas y sus resguardos y las comunidades afrocolombianas.

De hecho, para Díaz y Sánchez (2004), la expansión y escalamiento del conflicto armado durante la década de los 
noventa tiene un significativo efecto sobre el aumento de la producción de hoja de coca en Colombia. De igual forma, Botero (2013) muestra que el endurecimiento de la política antidrogas en Colombia incrementa la tasa de homicidios y el desplazamiento forzado durante el periodo de 1999 a 2010.

Por otra parte, recientemente Peter Reuter (2014) llamó la atención sobre la influencia del efecto globo en el análisis de la movilidad de las redes de tráfico de drogas. En su artículo, este autor presenta una definición general y precisa de la hipótesis del efecto globo sobre la manera como las actividades de producción y tráfico de drogas ilegales se reacomodan ante la represión ejercida por las autoridades de control en las diferentes etapas de la cadena productiva, principalmente a través de la implementación de diferentes planes de interdicción a los cargamentos de drogas ilegales o de erradicación a los cultivos ilegales:

Planteándolo de manera simple, esta hipótesis establece que si las autoridades endurecen la represión a la producción, el tráfico o el comercio en un lugar, entonces la actividad objetivo se desplazará a otro lugar, generando no más que dificultades temporales a los participantes. Las consecuencias de largo plazo, en términos de la disponibilidad de las sustancias ilícitas y sus precios para los consumidores, serán solo leves, especialmente cuando la intervención se ha dado cerca a los lugares de producción (Reuter, 2014, p. 33)2.

En este artículo el análisis se concentra en el cumplimiento de los efectos globo en la etapa de la cadena de producción de cocaína correspondiente a los cultivos ilegales. Sin embargo, la presencia de este tipo particular de efectos globo también tiene implicaciones para el estudio de otro tipo de efectos globo en otras fases de la cadena productiva. Lo último corresponde a un tema que merece ser tratado en próximos estudios en la materia.

\section{Justificación de la investigación}

En vista de que ninguno de los trabajos desarrollados hasta ahora en la materia, ha hecho un análisis econométrico para poner a prueba la presencia simultánea de efectos globo en los tres principales países productores de cocaína a lo largo del periodo 1990-2009, éste trabajo se propone llenar un vacío en el estudio de los cultivos ilegales, aplicando un modelo de ecuaciones estructurales -un modelo SUR- que logra captar de manera apropiada las interacciones propias de los procesos de relocalización de los cultivos ilegales en la Región Andina. En ese sentido la contribución

La traducción es nuestra. 
de este trabajo es doble: en primer lugar, se expone un análisis conceptual y descriptivo sobre el efecto globo a lo largo del periodo correspondiente a las dos décadas anteriores, el cuál es útil para entender la evolución de los cultivos con coca en la Región Andina. En segundo lugar, se pone prueba estadísticamente la hipótesis de que $a$ lo largo del periodo de estudio se han presentado efectos globo en la Región Andina. Esto último permite actualizar y ampliar el análisis realizado para el periodo 1987-2001 por parte de Moreno-Sánchez et al. (2003); pero la novedad de nuestro trabajo es la utilización de un modelo de carácter estructural como el SUR. Adicionalmente, estas contribuciones permiten evaluar la eficacia de las políticas de erradicación de los cultivos a lo largo del periodo de estudio, lo que es de gran importancia en la actualidad para evaluar los resultados de la política antidrogas y, en particular, del Plan Colombia durante las décadas anteriores; esto puede hacerse en la actualidad debido a que hay mayor claridad sobre la evolución de los cultivos de coca, la producción de cocaína y sus precios durante las dos décadas anteriores.

\section{ANÁLISIS DESCRIPTIVO}

Hay una evidencia descriptiva suficiente de que se ha cumplido la presencia de efectos globo para los cultivos de hoja de coca y la producción potencial de hoja de coca seca en la región andina a lo largo del periodo observado.
Durante el periodo de 1990 a 2010 se presentó un descenso significativo en el número de hectáreas cultivadas con coca para los tres principales productores (Colombia, Perú y Bolivia). Se pasó de cultivar 211.700 ha en 1990 a 158.000 ha en el año 2010 para los tres países andinos, lo que significa una reducción de $25 \%$ en los cultivos de coca para ese periodo a una tasa promedio anual de $-1.45 \%$ (UNODC, 1999, 2010 y 2011). Pero es necesario dividir el análisis temporal para identificar dos patrones de comportamiento diferentes a partir de la incidencia de las políticas de represión a la oferta y la evolución de las condiciones sociales, económicas y políticas.

El primer periodo de tiempo corresponde a toda la década de los noventa, periodo en que creció el número total de hectáreas de los tres países andinos en 9.600 ha, hasta alcanzar las 221.300 ha en el año 2000. Esto representa un crecimiento del $4.5 \%$ entre estos dos años de referencia y una tasa de crecimiento implícita de $0,44 \%$ anual para ese mismo periodo. No obstante, la participación de los países dentro del total de hectáreas cultivadas cambió en el transcurso de esta década, como se puede apreciar en la Figura 1. En el caso de los cultivos en Colombia se observa un aumento del $300 \%$, pasándose de cultivar 40.100 ha al comienzo del periodo a 163.300 ha al final, lo que representa una tasa de crecimiento implícita del $14 \%$ anual. Mientras que el número de hectáreas cultivadas en Perú 
y Bolivia bajó: en el primer país a una tasa implícita de $-12,4 \%$ anual, y en el segundo a una tasa de $-10,3 \%$ anual (cálculos propios a partir de la información de UNODC, 1999, 2010 y 2011).

La literatura describe algunos factores que explican el auge y crecimiento de los cultivos ilícitos en Colombia durante la década de los noventa. El primero es la expansión del conflicto armado irregular en Colombia que se reseña en trabajos como los de Díaz y Sánchez (2004), Posada y Mejía (2007), entre otros. Estos trabajos aducen que después del desmantelamiento de los dos grandes carteles en el primer lustro de la década de los noventa, adquirieron mayor preponderancia las guerrillas y las autodefensas en el manejo de la producción y comercialización de cocaína. Así, los grandes grupos armados ilegales potenciaron su capacidad para financiar sus acciones insurgentes y paramilitares $\mathrm{y}$, de esa manera, lograron amplificar su poderío militar y habilidades logísticas para controlar más vastos territorios.

En segundo lugar, durante la década de los noventa se presentó una consolidación y ampliación de la frontera agrícola dedicada al cultivo de coca en Colombia. Los campesinos víctimas de la crisis agraria ocasionada por la pérdida de competitividad del café colombiano y las políticas de apertura económica implementadas en Colombia, vieron en los cultivos ilícitos una alternativa para sus menguados beneficios. Los campesinos expandieron el territorio de cultivos de coca y se organizaron políticamente para defender sus territorios de las fumigaciones, aunque con limitada independencia de los actores armados, especialmente de la guerrilla (Thoumi, 2009).

En tercer lugar, luego de la desarticulación de los carteles de la droga como el de Medellín y el de Cali, se presentó una reestructuración de la producción y comercialización de cocaína en Colombia. Durante esta década emergió un número importante de nuevas organizaciones dedicadas al comercio ilegal de droga que tuvieron que contratar a las guerrillas y autodefensas para el mantenimiento de sus sistemas de seguridad y la provisión de insumos para el procesamiento de cocaína (Thoumi, 2009). Estos narcotraficantes ampliaron su trabajo político y desarrollaron alianzas con la clase política para defender sus intereses, tanto a nivel local como a nivel nacional (Duncan, 2014).

En cuarto lugar, el incremento de las plantaciones en Colombia durante este periodo también es atribuido a la fuerte represión ejercida en Perú durante el Gobierno de Fujimori (Thoumi, 2009). Desde 1995 fue establecida la estrategia del derrumbe del puente aéreo con Colombia, que consistió en el derribo e interceptación de aeronaves cargadas con coca desde el Perú. De igual forma, durante la primera mitad de la 
década de los noventa los precios de la coca en las principales regiones productoras de coca del Perú descendieron y los productores se vieron forzados a contribuir con rentas exógenas a las corruptas fuerzas de policía y agentes gubernamentales (Thoumi, 2009).

Por último, en el caso de Bolivia se implementó el "Plan Dignidad", que redujo los cultivos de forma acelerada a finales de la década de los noventa en ese país. Este Plan consistió en la reducción gradual de las compensaciones por eliminación de cultivos de coca durante el segundo Gobierno de Banzer (1997-2001) (Laserna, 2009).

El segundo periodo corresponde a la primera década del siglo XXI. En este sucedió lo contrario a los patrones de comportamiento de los cultivos ilícitos en los países andinos durante los años noventa. La cantidad total de hectáreas cultivadas con coca en Colombia, Perú y Bolivia bajó entre el año 2000 y el año 2010, pasando de 221.300 hectáreas a 158.000. Esta reducción de 63.300 hectáreas equivale a una reducción del 28 $\%$ entre esos dos referentes de tiempo y representa tasa de crecimiento implícita anual promedio cercana a $-3.4 \%$ para ese mismo periodo.

Para el caso de Colombia el número de hectáreas cultivadas bajó, pasando de 163.300 al comienzo del periodo, a 62.000 en el año 2010. Esto corresponde a una reducción de más de
101.000 hectáreas, que equivale a un pérdida de $62 \%$ del área de cultivos de coca entre estos dos años. Esto significa una reducción promedio anual del $9.23 \%$, resultado del fortalecimiento de la política antidrogas con los distintos componentes del "Plan Colombia", según algunos autores como Posada y Mejía (2007). Con esta estrategia múltiple se intensificó la erradicación y la fumigación aérea en las regiones cocaleras utilizando imágenes satelitales para focalizar y localizar los cultivos con mayor eficiencia. De esta forma, Colombia pasó de contribuir con en el $73 \%$ del área cultivada de coca a solo el $39 \%$ durante el periodo de tiempo referenciado (2000-2010).

Por su parte, el número de hectáreas cultivadas en Perú y Bolivia se incrementó. En Perú éste pasó de 43.400 ha en el año 2000 a 61.200 ha en el año 2010. Entre tanto, en Bolivia éste se duplicó pasando de 14.600 ha al comienzo del periodo a 30.900 al final. Estos incrementos en Perú y Bolivia sugieren que la estrategia de represión a la oferta, los recursos y la atención se localizó en Colombia (Bagley, 2012).

En Bolivia se llevaron a cabo varias protestas y revueltas cocaleras a principios del siglo XXI, debido al impacto económico y social de la erradicación de los cultivos de coca dentro del marco del Plan Dignidad (Laserna, 2009). A partir de ese momento los cultivos empezaron a crecer nuevamente y con 
la llegada del Gobierno de Evo Morales se planteó un reto: luchar contra el narcotráfico, sin erradicar los cultivos de coca y sin ejercer represión a los cocaleros (Laserna, 2009). Con este propósito se formuló la "Estrategia de lucha contra el narcotráfico y la revalorización de la coca" para el periodo 2007 a 2010, la cual tenía dos ejes de acción: uno represivo contra el narcotráfico y sus delitos conexos, y otro de control social y aprovechamiento de los cultivos de coca que se pretendían estabilizar en 20.000 ha (Laserna, 2009).

En el Perú se estabilizaron los cultivos de coca a partir de 1998 por una serie de factores claves (Soberón, 2009). Para Soberón, desde ese año se restablecieron los cultivos de los valles cocaleros peruanos, porque se ampliaron las actividades colaterales a este negocio ilícito, como las facilidades de servicios de transporte de coca, cocaína e insumos, $\mathrm{y}$ se fragmentaron los terrenos dedicados al cultivo de hoja de coca en parcelas mucho más pequeñas y escondidas donde se hace difícil la erradicación y fumigación (Soberón, 2009). Además, el Gobierno peruano dejó en el completo abandono institucional a las regiones cocaleras, permitiendo el establecimiento de estructuras económicas y sociales de índole criminal y corruptas que alejan un desarrollo sustentable de la tierra en el Perú (Soberón, 2009).

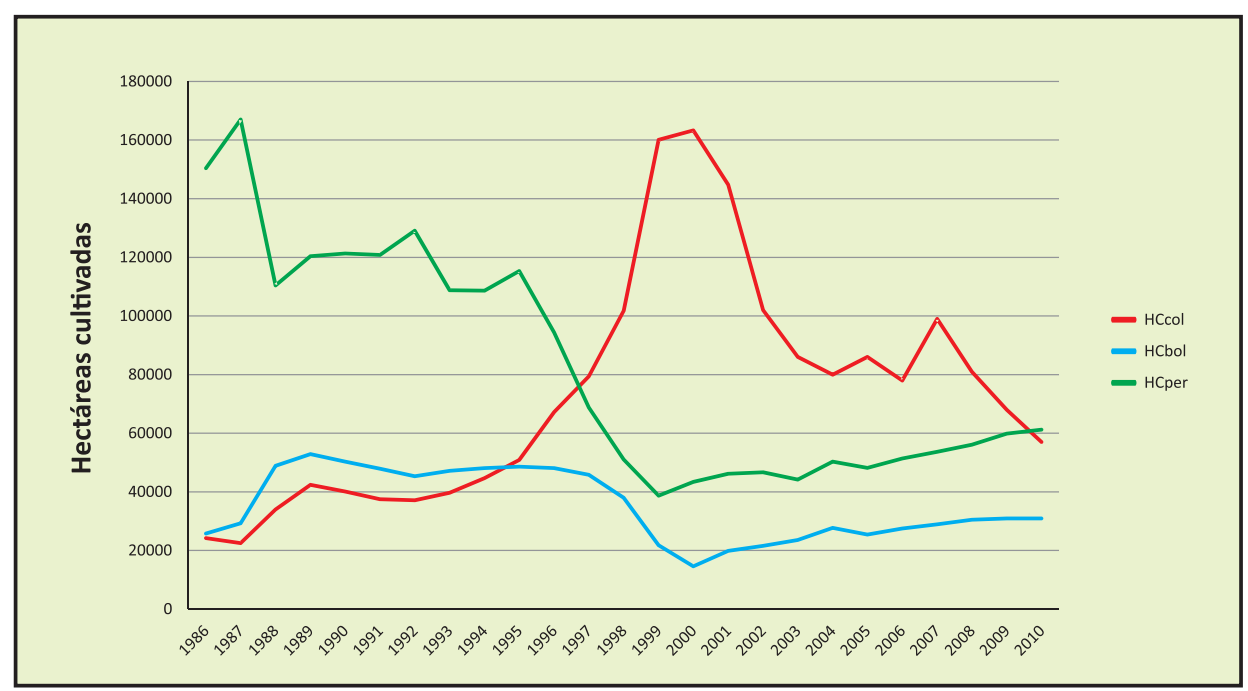

Figura 1. Hectáreas de coca cultivadas por países $(\mathrm{HC})$ Fuente: 1986-1987: (UNODC, 1988); 1990-1994: (UNODC, 2001); 1995-2010: (UNOCD, 2010, 2011).

3 En adelante se hará referencia a toda la información del sistema de información de UNOCD mencionando solo a UNOCD. 
De igual manera, puede observarse que se cumple el efecto globo para la producción de hoja de coca, tal como se puede apreciar en la Figura 2.

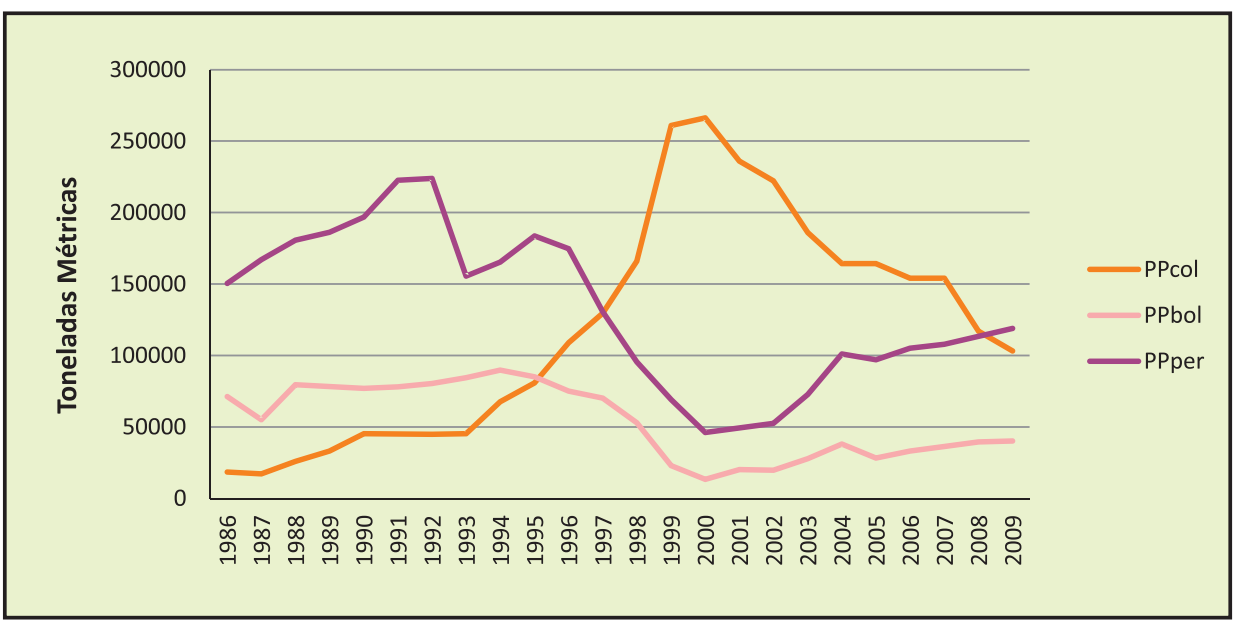

Figura 2. Producción potencial de hoja de coca por países (PP) Fuente: 1986-1987: (UNODC, 1988); 1990-1994: (UNODC, 2001); 1995-2010: (UNOCD, 2010, 2011).

La anterior descripción temporal de la evolución de los cultivos de coca revela que durante el periodo 1990-2000 los cultivos crecieron en Colombia a la vez que bajaron en Perú y Bolivia. En cambio, durante la década siguiente sucedió lo contrario: bajaron en Colombia al tiempo que crecieron y se estabilizaron en Perú y Bolivia. La Figura 1 ilustra este comportamiento: aquí se observa contundentemente que las tendencias en Colombia se yuxtaponen a las tendencias observadas en Perú y Bolivia.

\section{METODOLOGÍA}

Todas las estadísticas proceden de una base de datos organizada con estadísticas extraídas en su totalidad del sistema de información de UNOCD, el cual a su vez utiliza información del Sistema Integrado de Monitoreo de Cultivos Ilícitos (SIMCI) ${ }^{3}$. Debido a que la medición de las estadísticas de producción potencial de la hoja de coca que realiza UNOCD se basa en una serie de estimaciones realizadas a partir de la medición de hectáreas cultivadas con coca, el análisis estadístico que se presenta a continuación se concentra en el estudio de los determinantes de las hectáreas cultivadas con coca a lo largo del periodo 1990-2009.

La lista de variables utilizadas, junto con su definición y signos esperados en la estimación, se encuentra en la Tabla 1. Cabe anotar que la variable 
Los efectos globo en los cultivos de coca en la Región Andina (1990-2009)

Leonardo Raffo López • Javier Andrés Castro • Alexander Díaz España

de proporción de la producción agregada agrícola con respecto al PIB para Bolivia se extrajo de las estadísticas del Banco Mundial.

Tabla 1. Descripción de las variables del sistema SUR

\begin{tabular}{|c|c|c|}
\hline $\begin{array}{l}\text { Periodo: } 1991- \\
2009\end{array}$ & Mercado: produccion de coca & Metodologia: SUR \\
\hline Variables & Definición & Signo esperado \\
\hline Inhccol $_{t}$ & $\begin{array}{l}\text { Logaritmo natural del área cultivada con coca en Colombia } \\
\text { (ha). Fuente: UNODC (1986-1998 Global Illicit Drug Trends } \\
\text { 1999; 1999-2009 World Drug Report 2010). }\end{array}$ & $\begin{array}{l}\text { No aplica, variable } \\
\text { dependiente }\end{array}$ \\
\hline $\operatorname{Inhccol}_{\mathrm{t}-1}$ & $\begin{array}{l}\text { Logaritmo natural del área cultivada con coca rezagada un pe- } \\
\text { riodo en Colombia (ha). Fuente: UNODC (1986-1998 Global Illi- } \\
\text { cit Drug Trends 1999; 1999-2009 World Drug Report 2010). }\end{array}$ & Positivo \\
\hline Inp1usa09 & $\begin{array}{l}\text { Logaritmo natural del precio en dólares US al por menor de } \\
\text { coca/gramo por niveles de pureza ajustado por inflación de } \\
2009 \text { para USA 1990-2009. Fuente: World Drug Report } 2011 .\end{array}$ & Positivo \\
\hline Inhcbolper $_{t}$ & $\begin{array}{l}\text { Logaritmo natural del área cultivada con coca en Bolivia y } \\
\text { Perú (ha). Fuente: UNODC (1986-1998 Global Illicit Drug } \\
\text { Trends 1999; 1999-2009 World Drug Report 2010). }\end{array}$ & Negativo \\
\hline Inecol $_{(\mathrm{t}-1)}$ & $\begin{array}{l}\text { Logaritmo natural de erradicación de cultivos con coca pa- } \\
\text { ra Colombia (ha). Fuente: UNODC (1986-1998 Global Illicit } \\
\text { Drug Trends 1999; 1999-2009 World Drug Report 2010). }\end{array}$ & Negativo \\
\hline dummycol & $\begin{array}{l}\text { Variable dummy que capta el cambio estructural que provo- } \\
\text { có el Plan Colombia a partir del año } 2001\end{array}$ & Negativo \\
\hline Inedplan & $\begin{array}{l}\text { Variable dummy interactiva entre el logaritmo de la erradica- } \\
\text { ción rezagada y el cambio estructural que provocó el Plan } \\
\text { Colombia. }\end{array}$ & Positivo \\
\hline Inhcbol & $\begin{array}{l}\text { Logaritmo natural del área cultivada con coca en Bolivia } \\
\text { (ha). Fuente: UNODC (1986-1998 Global Illicit Drug Trends } \\
\text { 1999; 1999-2009 World Drug Report, 2010). }\end{array}$ & $\begin{array}{l}\text { No aplica, variable } \\
\text { dependiente }\end{array}$ \\
\hline Inhcbol $_{(\mathrm{t}-1)}$ & $\begin{array}{l}\text { Logaritmo natural del área cultivada con coca rezagada un pe- } \\
\text { riodo en Bolivia (ha). Fuente: UNODC (1986-1998 Global Illi- } \\
\text { cit Drug Trends 1999; 1999-2009 World Drug Report 2010). }\end{array}$ & Positivo \\
\hline Inebol $_{(\mathrm{t}-1)}$ & $\begin{array}{l}\text { Logaritmo natural de erradicación de cultivos con coca para } \\
\text { Bolivia (ha). Fuente: UNODC (1986-1998 Global Illicit Drug } \\
\text { Trends 1999; 1999-2009 World Drug Report 2010). }\end{array}$ & Negativo \\
\hline dummybol & $\begin{array}{l}\text { Dummy que capta el cambio estructural que provocó el plan } \\
\text { de represión antidroga del presidente Banzer para los años } \\
\text { 1998-2000. }\end{array}$ & Negativo \\
\hline Inedbol & $\begin{array}{l}\text { Variable dummy interactiva entre el logaritmo de la erradica- } \\
\text { ción rezagada y el cambio estructural que provocó el plan } \\
\text { de represión antidroga del presidente Banzer. }\end{array}$ & Positivo \\
\hline
\end{tabular}


Apuntes CENES Volumen 35, Número 61, ISSN 0120-3053

enero - junio 2016, Págs. 207-236

\begin{tabular}{|c|c|c|c|}
\hline $\begin{array}{l}\text { Periodo: } 1991- \\
2009\end{array}$ & Datos: series de tiempo & Mercado: produccion de coca & Metodologia: SUR \\
\hline Variables & \multicolumn{2}{|c|}{ Definición } & Signo esperado \\
\hline ppibagrbol $_{t}$ & \multicolumn{2}{|c|}{$\begin{array}{l}\text { Proporción del valor agregado del sector agrícola en el PIB } \\
\text { total para Bolivia. Fuente: Banco mundial. }\end{array}$} & Positivo \\
\hline Inhccolper $_{t}$ & \multicolumn{2}{|c|}{$\begin{array}{l}\text { Logaritmo natural del área cultivada con coca en Colombia } \\
\text { y Perú (ha). Fuente: UNODC (1986-1998 Global Illicit Drug } \\
\text { Trends 1999; 1999-2009 World Drug Report 2010). }\end{array}$} & Negativo \\
\hline Inhcper $_{t}$ & \multicolumn{2}{|c|}{$\begin{array}{l}\text { Logaritmo natural del área cultivada con coca en Perú (ha). } \\
\text { Fuente: UNODC (1986-1998 Global Illicit Drug Trends 1999; } \\
\text { 1999-2009 World Drug Report 2010). }\end{array}$} & $\begin{array}{l}\text { No aplica, variable } \\
\text { dependiente }\end{array}$ \\
\hline Inhcper $_{(\mathrm{t}-1)}$ & \multicolumn{2}{|c|}{$\begin{array}{l}\text { Logaritmo natural del área cultivada con coca rezagada un } \\
\text { periodo en Perú (ha). Fuente: UNODC (1986-1998 Global } \\
\text { Illicit Drug Trends 1999; 1999-2009 World Drug Report } \\
\text { 2010). }\end{array}$} & Positivo \\
\hline Ineper $_{(\mathrm{t}-1)}$ & \multicolumn{2}{|c|}{$\begin{array}{l}\text { Logaritmo natural de erradicación de cultivos con coca pa- } \\
\text { ra Perú (ha). Fuente: UNODC (1986-1998 Global Illicit Drug } \\
\text { Trends 1999; 1999-2009 World Drug Report 2010). }\end{array}$} & Negativo \\
\hline dummyper & \multicolumn{2}{|c|}{$\begin{array}{l}\text { Variable dummy que capta el cambio estructural que provo- } \\
\text { có el periodo de represión de Fujimori (1991-2000) }\end{array}$} & Negativo \\
\hline Inedper & \multicolumn{2}{|c|}{$\begin{array}{l}\text { Variable dummy interactiva entre el logaritmo de la erradica- } \\
\text { ción rezagada y el cambio estructural que provocó el perio- } \\
\text { do de represión de Fujimori. }\end{array}$} & Positivo \\
\hline Inhccolbol & \multicolumn{2}{|c|}{$\begin{array}{l}\text { Logaritmo natural del área cultivada con coca en Colom- } \\
\text { bia y Bolivia (ha). Fuente: UNODC (1986-1998 Global Illicit } \\
\text { Drug Trends 1999; 1999-2009 World Drug Report 2010). }\end{array}$} & Negativo \\
\hline
\end{tabular}

Fuente: base de datos construida a partir de la información de UNOCD.

Para este trabajo se especificó y estimó un conjunto de regresiones correspondiente a un modelo SUR, que busca examinar empíricamente algunos de los determinantes de las hectáreas cultivadas con coca en Colombia, Perú y Bolivia y, en particular, poner a prueba la hipótesis de que a lo largo del periodo 1990-2009 se han presentado fuertes efectos globo en el territorio geográfico correspondiente a los principales países productores de cocaína en la región andina.
Este modelo es pertinente porque captura las posibles correlaciones en los términos de error entre las regresiones que explican el número de hectáreas cultivadas para los tres principales productores, lo que en este caso es clave debido a la presencia del efecto globo y de las interrelaciones derivadas de la integración de la cadena productiva de los negocios ilegales entre los tres países. Dado el carácter geográfico de las variables estudiadas en la presente investigación, las hectáreas 
cultivadas con coca en Colombia, Bolivia y Perú pueden estar correlacionadas entre sí y, a su vez, pueden existir factores que no están contemplados en las especificaciones que están correlacionados con factores de Colombia, Bolivia y Perú. Es decir, las hectáreas cultivadas de un país productor de cocaína pueden estar correlacionadas con las hectáreas cultivadas de coca de los productores que se encuentran geográficamente cerca. Hasta ahora ningún trabajo en la materia había utilizado el modelo SUR para examinar el efecto globo para toda la Región Andina durante el periodo 1990-2009. Ésta es por ende la principal contribución de este trabajo. Próximos trabajos debería actualizar el modelo para determinar lo que ha acontecido con las dinámicas de los cultivos de coca en la región durante la última década.

Como es usual, el modelo SUR se estimó mediante el método de mínimos cuadrados generalizados (MCG) para lograr una estimación más eficiente que por mínimos cuadrados ordinarios (MCO) (ver Greene, 2008) y se consideraron los siguientes supuestos de partida sobre la estructura de la matriz de covarianzas:

1) En cada ecuación del sistema SUR el término de error no presenta ni autocorrelación ni heterocedasticidad.

2) La correlación entre los errores de cada especificación del sistema SUR es contemporánea, es decir:

$$
E\left(U_{j} U_{i}\right)=\sigma_{i j} I_{T} \quad i \neq j \quad i, j=1,2,3, \ldots, m,
$$

en donde $m$ es el número de ecuaciones del modelo -en nuestro caso 3 ecuaciones, una para cada uno de los principales productores de la región (Bolivia, Colombia y Perú) y T el número de observaciones.

Usando una notación matricial el modelo se puede representar así:

$$
\mathbf{H C}=\mathbf{X \beta}+\mathbf{U}, \quad[1]
$$

en donde $\mathbf{H C}$ es el vector de hectáreas cultivadas con coca de dimensión $T \cdot 3 \times 1, \mathbf{X}$, es una matriz de dimensión $T \cdot 3 \times \sum_{i=1}^{3} K_{i}, \boldsymbol{B}$ es el vector de coeficientes de dimensión $\sum_{i=1}^{3} K_{i}$ y $\mathbf{U}$ es el vector de errores aleatorios de dimensión $T \cdot 3 \times 1$. El modelo también se puede representar más detalladamente así:

$$
\left[\begin{array}{l}
\mathrm{HC}_{C o l} \\
\mathrm{HC}_{B o l} \\
\mathrm{HC}_{P e r}
\end{array}\right]=\left[\begin{array}{ccc}
\mathrm{X}_{C o l} & 0 & 0 \\
0 & \mathrm{X}_{B o l} & 0 \\
0 & 0 & \mathrm{X}_{P e r}
\end{array}\right]+\left[\begin{array}{c}
\mathrm{U}_{C o l} \\
\mathrm{U}_{B o l} \\
\mathrm{U}_{P e r}
\end{array}\right]
$$

En este sistema de ecuaciones estructurales cada ecuación constituye una regresión clásica; por tal razón los parámetros podrían ser estimados consistentemente pero no eficientemente utilizando MCO para cada ecuación por separado (Greene, 2008). La matriz de varianzas covarianzas es $\Omega=\Sigma \times \mathrm{I}$ en donde

$$
\Sigma=\left[\begin{array}{lll}
\sigma_{11} & \sigma_{12} & \sigma_{13} \\
\sigma_{21} & \sigma_{22} & \sigma_{23} \\
\sigma_{31} & \sigma_{32} & \sigma_{33}
\end{array}\right]
$$

En este caso el estimador MCO ignora la información contenida en las 
covarianzas entre observaciones contemporáneas de distintas ecuaciones. Este comportamiento solo sería eficiente en el caso de que dichas covarianzas fuesen cero. Por tanto, se usa la metodología de los MCG para que las estimaciones sean eficientes (Greene, op. cit.).

En el sistema de ecuaciones, un choque capturado por el término de error de una de las ecuaciones de alguno de los tres países puede estar afectando la oferta de coca de los demás países. El sistema de ecuaciones SUR viene dado por:

$$
\begin{gathered}
\text { Inhccol }=\alpha_{0}+\alpha_{1} \text { lnhccol }_{(t-1)}+\alpha_{2} \\
\text { Inp1usa09 }+\alpha_{3} \text { Inhcbolper }_{t}+ \\
\alpha_{4} \text { lnecol }_{(t-1)}+\alpha_{5} \text { lnedplan } \\
+\alpha_{6} \text { dummycol }+\varepsilon_{t}
\end{gathered}
$$

$$
\begin{gathered}
\text { Inhcbol }_{t}=\beta_{0}+\beta_{1} \text { lnhcbol }_{(t-1)}+\beta_{2} \\
\text { lnplusa09 }_{t}+\beta_{3} \text { Inhccolper }_{t}+\beta_{4} \\
\text { ppibagrbol }_{t}+\beta_{5} \text { lnebol }_{(t-1)}+\beta_{6} \\
\text { lnedbol }+\beta_{7} \text { dummybol }+\varepsilon_{t}
\end{gathered}
$$

$$
\begin{gathered}
\text { lnhcper }_{t}=\gamma_{0}+\gamma_{1} \text { Inhcper }_{(t-1)} \\
+\gamma_{2} \text { Inplusa09 }_{t}+\gamma_{3} \text { Inhccolbol }_{t} \\
+\gamma_{4} \text { lneper }_{(t-1)}+\gamma_{5} \text { lnedper } \\
+\gamma_{6} \text { dummyper }+\varepsilon_{t}
\end{gathered}
$$

Cabe agregar que con series de tiempo más largas podría ser interesante utilizar técnicas de series de tiempo como el análisis de intervención para series de tiempo multivariadas. No obstante, con lo datos disponibles para el periodo de estudio las series con cortas, por lo que nos parece más apropiado utilizar un modelo de carácter estructural como el SUR. Este modelo es el más apropiado para examinar la presencia simultánea de efectos globo para los tres países suramericanos. Futuros trabajos para periodos de estudio más extensos podrían explorar métodos de series de tiempo.

\section{RESULTADOS}

En la Tabla 2 se presentan los resultados del modelo SUR con variable dependiente hectáreas cultivadas para los principales países cultivadores de planta de hoja de coca en Suramérica: Colombia, Bolivia y Perú. Las variables se encuentran en logaritmos, a excepción de la participación del PIB agrícola para Bolivia en el producto total, para una mejor interpretación. En cada ecuación -para cada país- se ha incluido una variable que capta la incidencia de efectos globo: para Colombia esta corresponde a la variable lnhbolper, que mide la suma de las hectáreas cultivadas en Perú y Bolivia (en logaritmos); para Bolivia esta corresponde a la variable lnhcolper, que mide la suma de las hectáreas cultivadas en Perú y Colombia (en logaritmos); para Perú corresponde a la variable lnhcolbol, la cual mide la suma de las hectáreas cultivadas en Bolivia y Colombia (en logaritmos). Como 
lo mencionamos antes, en su modelo Moreno-Sánchez et al. (2003) incorporan una variable análoga para captar el efecto globo en el caso las hectáreas cultivadas con coca en Colombia en función de los cultivos en los otros dos países de la región.

El test de Breusch-Pagan de independencia de los errores de las ecuaciones indica que los errores están correlacionados, por lo que la metodología SUR es adecuada para el análisis de los cultivos de coca en Colombia, Bolivia y Perú. Por otra parte, la matriz de correlaciones de los errores muestra que los cultivos de planta de coca en Colombia y Perú están altamente correlacionados y que los cultivos de planta de coca en Bolivia no se correlacionan significativamente con los mercados de Colombia y Perú.

Tabla 2. Modelo SUR con variables de efecto globo

\begin{tabular}{|c|c|c|c|c|c|c|c|c|}
\hline Variables & Inhccol $_{\mathrm{t}}$ & Inp1usa09, & Inhcbolper $_{t}$ & & Inecol $_{(t-1)}$ & dummycol & Inecdplan & $\mathrm{R}^{2}$ \\
\hline $\begin{array}{c}\text { Ecuación } \\
{[4]}\end{array}$ & endógena & $\begin{array}{c}0.282^{* *} \\
(0.124)\end{array}$ & $\begin{array}{c}-1.06^{* * *} \\
(0.093)\end{array}$ & & $\begin{array}{c}0.090 * * * \\
(0.019)\end{array}$ & $\begin{array}{c}2.554^{* * *} \\
(0.836)\end{array}$ & $\begin{array}{c}-0.025^{\star * *} \\
(0.071)\end{array}$ & 0.95 \\
\hline Variables & Inhcbol & Inp1usa09, & Inhccolper $_{t}$ & ppibagrbol $_{t}$ & Inebol $_{(t-1)}$ & dummybol & Inecdpol & $\mathrm{R}^{2}$ \\
\hline $\begin{array}{c}\text { Ecuación } \\
{[5]}\end{array}$ & endógena & $\begin{array}{c}0.391 * * \\
(0.267) \\
\end{array}$ & $\begin{array}{c}-0.842^{*} \\
(0.467) \\
\end{array}$ & $\begin{array}{c}16.307^{* * *} \\
(3.740) \\
\end{array}$ & $\begin{array}{c}-0.158^{\star} \\
(0.087) \\
\end{array}$ & $\begin{array}{l}12.469 \\
(8.452) \\
\end{array}$ & $\begin{array}{l}-1.334 \\
(0.891) \\
\end{array}$ & 0.75 \\
\hline Variables & Inhcper $_{t}$ & Inp1usa09, & Inhccolbol & & Ineper $_{(t-1)}$ & dummyper & Inecdper & $\mathrm{R}^{2}$ \\
\hline $\begin{array}{c}\text { Ecuación } \\
{[6]}\end{array}$ & endógena & $\begin{array}{c}0.396^{\star \star \star} \\
(0.168)\end{array}$ & $\begin{array}{c}-1.334^{* \star *} \\
(0.120)\end{array}$ & & $\begin{array}{l}-0.158 \\
(0.127)\end{array}$ & $\begin{array}{l}-1.184 \\
(1.263)\end{array}$ & $\begin{array}{c}0.173 \\
(0.139)\end{array}$ & 0.90 \\
\hline \multicolumn{5}{|c|}{ Test de independencia de Breusch-Pagan } & \multicolumn{4}{|c|}{$X^{2}=9.757^{\star \star}$} \\
\hline \multicolumn{9}{|c|}{ Matriz de correlación de residuales } \\
\hline & Inhccol $_{t}$ & Inhccol & Inhcper $_{t}$ & & & & & \\
\hline Inhccol $_{t}$ & 1.0000 & & & & & & & \\
\hline Inhccol $_{t}$ & 0.2110 & 1.0000 & & & & & & \\
\hline Inhcper $_{t}$ & 0.6812 & 0.0704 & 1.0000 & & & & & \\
\hline
\end{tabular}

*Los errores estándar se encuentran entre paréntesis.

$*<0.1 ; * *<0.05 ; * * *<0.01$

Fuente: cálculos propios con base en las variables construidas.

Los resultados indican que un aumento del precio de la cocaína al por menor en Estados Unidos conduce a un aumento del cultivo de planta de coca en los tres países. Esto se explica por los incentivos para incrementar la producción, que se generan por un incremento en las ganancias esperadas de los traficantes como resultado del incremento en los precios en Estados Unidos, y lo contrario cuando los precios bajan. Este efecto puede concebirse como un efecto directo o de oferta de los precios de los estupefacientes en los lugares de consumo sobre la producción de cocaína y de hoja 
de coca en los países productores. La razón por la que el precio al por menor del alcaloide influye en las hectáreas cultivadas con coca en los países productores es que toda la cadena productiva mantiene un nivel relativamente alto de integración vertical bajo el mando de los grandes traficantes de drogas, cuyos incentivos $-\mathrm{y}$, por ende, el de los agentes que participan en los extremos de la cadena- se regulan por la evolución de las ganancias del negocio, cuya dinámica en últimas depende del comportamiento de los precios en los grandes centros de consumo ${ }^{4}$.

Según este resultado, puede decirse que la tendencia a largo plazo a la baja en los precios de la cocaína -tanto al por mayor como al por menorque se presentó en Estados Unidos a lo largo de todo el periodo de estudio (UNOCD, 2011) ceteris paribus generó incentivos para que los productores de cocaína produjeran una menor cantidad de cocaína y, como consecuencia, que los campesinos cultivadores cultivarán menos coca.

En la cuarta columna de la Tabla 2 se encuentra la variable que capta la posible presencia de efectos globo en la muestra suramericana. Se observa que la variable es altamente significativa para las tres ecuaciones y exhibe el signo esperado, corroborando la existencia de procesos de relocalización de los cultivos de planta de coca en Colombia, Bolivia y Perú. Este es un resultado fundamental para probar la presencia de fuertes efectos globo entre estos tres países. Según nuestro modelo (ver la Tabla 2), un incremento de un punto porcentual en las hectáreas cultivadas con coca en Perú y Bolivia produce ceteris paribus una baja de más de $1,06 \%$ en las hectáreas cultivadas con coca en Colombia. Por otra parte, un incremento de un punto porcentual en las hectáreas cultivas con coca en Colombia y Perú ceteris paribus lleva a una disminución del 0,842 $\%$ en las hectáreas cultivadas con coca en Bolivia. Por último, un incremento de un punto porcentual en las hectáreas cultivadas con coca en Colombia y Bolivia ceteris paribus induce una disminución del $1,334 \%$ en las hectáreas cultivadas con coca en Perú.

Estos hallazgos implican una elasticidad unitaria (de $-1,06 \%$ ) de las

\footnotetext{
4 Cabe observar que en otro modelo estadístico distinto -al que por limitaciones de espacio no se hizo referencia en este trabajo- se encontró evidencia para el caso de Colombia de la presencia de otro tipo de efecto, esta vez un efecto indirecto en el sentido contrario del mencionado en el texto, que va de las hectáreas cultivadas a los precios de consumo: un mayor (menor) número de hectáreas cultivadas ceteris paribus conduce a un incremento (descenso) del precio de oferta en Colombia, lo que a su vez induce incrementos (disminuciones) en la producción de cocaína, que a la postre llevan a bajas (incrementos) en los precios de demanda en EE.UU.. En realidad los dos tipos de efectos contrapuestos no son contradictorios entre sí: el efecto directo puede concebirse como un efecto de oferta -de los precios al por menor en los lugares de consumo, en este caso EE.UU. a las hectáreas cultivadas con coca en los países productores-, mientras que el efecto indirecto puede concebirse como un efecto de demanda, que se desarrolla para el caso de Colombia, país que por ser el mayor productor de cocaína es el que mayor incidencia tiene en los precios en los grandes centros de consumo, en este caso el estadounidense.
} 
hectáreas cultivas con coca en Colombia frente a las de Perú y Bolivia, lo que confirma lo obtenido por Moreno-Sanchez et al. (2003) para Colombia durante el periodo 1988-2001. Por otra parte, la elasticidad de las hectáreas cultivadas en Perú frente a las de Colombia y Bolivia es mayor que 1 en valor absoluto (de -1,334\%); este resultado revela que las hectáreas cultivadas en este país son elásticas frente al comportamiento en las cultivadas en los otros dos países. Por último, para Bolivia las hectáreas cultivadas con coca también dependen del comportamiento de la misma variable para los otros dos países, aunque en este caso la elasticidad es menor que 1 pero cercana a éste valor (de -0,842\%). La menor elasticidad de las hectáreas cultivadas para Bolivia se debe a la estabilidad e importancia que tiene para ese país el consumo de hoja de coca con fines tradicionales.

La importancia económica de este hallazgo radica en que el efecto neto de la represión -y en particular, la erradicación- ejercida en cualquiera de los tres países tiende a ser nulo durante el periodo de análisis, debido a la rápida capacidad de las actividades ilegales para relocalizarse en los otros dos países. Es importante comprender, que el efecto globo no es una simple verdad de Perogrullo relacionada con el comportamiento de un mercado cualquiera, en donde con una demanda constante, una baja en la oferta por parte de algunos productores, llevaría a incrementos de la misma por parte de otros productores. En realidad se trata de un resultado determinante en el campo de la economía del crimen que debe ser entendido en el contexto de mercados ilegales sujetos a represión. El punto clave es que la represión a las drogas -en este caso la erradicación- no logra aminorar su oferta agregada, debido a la capacidad y versatilidad que tienen los agentes ilegales para reproducir sus actividades en otros contextos -espaciales, geográficos o incluso económicos en el caso de presentarse una diversificación de las actividades ilegales-, la cual depende a nivel micro de su capacidad para reaccionar de forma estratégica ante la represión ejercida por las autoridades de control (Raffo y Segura, 2015) ${ }^{5}$.

Así pues, los resultados obtenidos frente a la presencia de efectos globo son contundentes: estos juegan un papel esencial en el análisis y son determinantes de las hectáreas cultivadas con coca en los países productores.

Cabe agregar que en el caso de Colombia, aunque los cultivos bajaron según los datos de UNOCD durante el periodo 2000-2009, también hay

\footnotetext{
5 Raffo y Segura (2015) hacen un análisis del comportamiento estratégico de los narcotraficantes y otros agentes ligados a ellos, a través del cual se explica a nivel micro el llamado efecto hidra de las actividades ilegales (Ortiz, 2002. 2003, 2009), el cual está relacionado con el efecto globo. Ver más adelante las conclusiones de este artículo.
} 
evidencia del cumplimiento del efecto globo dentro del país: se presentaron procesos de relocalización geográfica de los cultivos de unas regiones del país a otras como resultado de las políticas de erradicación aplicadas (Thoumi, 2009). Esto llevó a una expansión de los cultivos a una mayor cantidad de departamentos del país, a pesar de la disminución en el número de hectáreas registradas con coca. En palabras de Thoumi: "Cuando bajo el Plan Colombia, que comenzó en 1999, se inició un método intensivo de erradicación, había plantaciones de coca en 12 departamentos. En 2003 se habían extendido a 23 de los 32 departamentos (UNOCD 2004:15). De manera simultánea, el tamaño promedio de las plantaciones ilegales descendió debido a que los campesinos buscaban evitar la fumigación aérea (Thoumi, 2009:85) ${ }^{6}$.

Por otra parte, la proporción del PIB agrícola en Bolivia incide de la forma esperada sobre las hectáreas cultivadas con coca y es altamente significativa. Este resultado se explica por la tradición de consumo de hoja de coca que existe en este país desde épocas remotas. En Bolivia, al igual que en otros países suramericanos, diversas comunidades reconocen que esta planta tiene propiedades curativas y medicinales valiosas. Esta costumbre tiene un fuerte arraigo cultural, político y económico en las comunidades indígenas, de origen indígena y mestizas de esta nación. Por consiguiente, este resultado muestra que los cultivos de coca en Bolivia han jugado un papel relevante en la agricultura de ese país durante las últimas décadas. Esto explica por qué los cocaleros han tenido una influencia determinante en la política en Bolivia, especialmente durante las últimas décadas. De ahí que la propuesta política del Gobierno de Evo Morales se haya concebido desde sus inicios como una agenda defensora de los derechos de los agricultores cocaleros en ese país. Aunque -como ya se mencionó en la tercera sección- como primer mandatario de Bolivia, Evo Morales planteó el reto de luchar contra el narcotráfico sin erradicar los cultivos de planta de coca ni vulnerar los derechos de los campesinos cocaleros (Laserna, 2009).

La variable de erradicación rezagada $\left(\operatorname{lnecol}_{\mathrm{t}-1}\right)$ es significativa para Colombia, pero no tiene el signo esperado. Lo que quiere decir que en términos netos - controlando por los otros factores considerados- la erradicación resulta ser contraproducente a lo largo de todo el periodo observado. Este resultado coincide con el efecto contraproducente de la erradicación hallado por Moreno-Sanchez et al. (2003) para Colombia durante el periodo 19872001. Este efecto contraproducente,

\footnotetext{
6 Próximos trabajados podrían examinar empíricamente la presencia de efectos globo en el interior del país utilizando modelos tipo panel.
} 
además, corrobora la presencia de efectos hidra (Ortiz, 2002, 2003 y 2009) que se explican a nivel micro por las reacciones estratégicas de los narcotraficantes ante la represión, las cuales hacen que ésta paradójicamente lleve a una reproducción de los negocios ilícitos y, por ende, de los cultivos ilícitos. Esto es consistente con el signo de los coeficientes de las variables dummy asociadas al Plan Colombia. A pesar de que la variable dummy con interacción (lnecdplan) es significativa $\mathrm{y}$ tiene un impacto negativo, la dummy de cambio estructural sin interacción (dummycol) es también significativa pero está asociada a un coeficiente positivo bastante alto. Esto corrobora algo evidente: el Plan Colombia mejoró le eficacia de la represión a las actividades a través de su interacción con la erradicación. Sin embargo, su impacto inicial fue contrarrestado por las fuerzas contrapuestas derivadas de los efectos globo y los efectos hidra.

De hecho, el signo positivo del coeficiente de la variable de erradicación rezagada confirma la importancia de los efectos globo para que las políticas de erradicación no tengan el efecto esperado para el caso de Colombia.

Para el caso de Bolivia la variable de erradicación rezagada $\left(\operatorname{lnebol}_{t-1}\right)$ sí tiene un impacto negativo, como era de esperarse, sobre las hectáreas cultivadas con coca, aunque esta variable solo resultó ser significativa al $10 \%$. Según las estimaciones se encuentra que un incremento de un punto porcentual en la erradicación produce ceteris paribus una disminución del 0,158 \% en el número de hectáreas cultivadas con coca en este país. En cambio las variables dummy no resultaron ser significativas. El mismo efecto tipo de efecto se mantiene después de controlar por los otros factores. No obstante, la falta de significancia de las variables dummy revela que en realidad la fuerte represión ejercida a finales de los años noventa a través del llamado "Plan Dignidad", durante el segundo periodo de presidencia de Hugo Bánzer, fue inútil. Esto es apoyado por Laserna (2009), quien aduce que la fuerte represión ejercida durante ese corto periodo realmente fue contraproducente, teniendo en cuenta lo que sucedió durante los años siguientes.

En cambio para Perú la variable de erradicación no resultó ser significativa. Tampoco la variable dummy. Este resultado implica que el impacto neto de la erradicación controlando por otros factores es irrelevante. Lo que indica el modelo estimado es que el efecto neto de la política de erradicación es nulo, de modo que en términos netos -controlando por los demás factores- la política de erradicación resulta ser ineficaz. Por lo tanto la baja en las hectáreas cultivadas durante los años noventa ciertamente no se explica por la represión ejercida por el Gobierno de Fujimori sino por otros factores. ¿Cuáles? Nuestro modelo también da luces al respecto: la baja de los precios de las drogas duras en Estados Unidos, 
por una parte, y la presencia de fuertes efectos globo con los otros dos países de la región, por otra parte.

Las estimaciones del modelo SUR son congruentes con la hipótesis de la interrelación de las economías ilegales de la muestra andina. Así, las estimaciones proporcionan evidencia a favor de la hipótesis, ya que existe un trade-off entre las distintas producciones de hoja de planta de coca en Colombia, Bolivia y Perú y la fuerte interdependencia entre las hectáreas cultivadas de Colombia y Perú. Pero hay algo más que decir sobre el modelo SUR: su buen ajuste también indica que hay otros factores no identificados aparte de los efectos globo que son captados por las correlaciones de los términos de error entre los tres países. ¿Qué clase de factores pueden ser éstos? Aquí puede jugar un papel crucial la integración vertical de toda la cadena de actividades ilegales alrededor de todo el territorio geográfico correspondiente a los tres países observados. Ésta se dio durante el periodo observado especialmente entre Colombia y Perú, ya que parte de la hoja de coca transformada en alcaloide en el territorio colombiano ha provenido -desde los inicios del tráfico de cocaína en los años setenta y a gran escala en los años ochenta- del Perú (Bagley, 1988).

Las tendencias observadas dan indicios de que estos procesos de tránsito de Perú a Colombia se volvieron a fortalecer durante la década pasada con la caída de los cultivos ilegales en
Colombia como resultado de la presión ejercida con el Plan Colombia. Esta mayor integración vertical de la cadena productiva entre Colombia y Perú se explica en parte por la mayor cercanía geográfica de Colombia con éste último. Pero, además, para el caso de Bolivia, hay indicios de que durante la década pasada se fortalecieron también las fases superiores de la cadena (de elaboración y procesamiento del alcaloide) (Laserna, 2009), permitiendo una mayor integración vertical de la economía ilegal dentro de este mismo país, o sea una menor integración vertical de la economía ilegal de ese país con las de los otros dos países. Prueba de ello es que la correlación parcial entre las hectáreas cultivadas de Colombia y Perú es más alta que las que se obtuvieron para Bolivia y Colombia, por una parte, y de Bolivia y Perú, por otra (de 0,6812 frente a 0,211 y 0,0704 respectivamente). Obsérvese también que, como era de esperarse, la correlación parcial de las hectáreas cultivadas de Colombia y Bolivia es considerablemente mayor que la hallada para las hectáreas de Perú y Bolivia. Además, en los tres casos las correlaciones parciales son positivas. Esto constata que el papel de Colombia en la cadena productiva es diferente del de los otros dos países, que no mantienen fuertes complementariedades de sus economías ilegales entre sí y se concentran en los primeros eslabones de la cadena.

Para completar el análisis se corrió de nuevo el modelo sin las variables 
interactivas de efecto globo. Tampoco se incluyeron las dummy interactivas para simplificar al máximo el sistema. Los resultados de este segundo modelo se presentan en la Tabla 3. Este nuevo sistema de ecuaciones también es robusto (según el test de Breusch-Pagan) y en general preserva los mismos resultados del modelo anterior; aunque ahora la variable de precio no es significativa para Colombia; adicionalmente, la variable dummy de cambio estructural para Bolivia se vuelve significativa y con un coeficiente asociado negativo.
La misma variable también se vuelve significativa pero con coeficiente asociado positivo para Perú. Pero hay una novedad sobresaliente entre este modelo y el primero: ahora las correlaciones parciales en la matriz de residuales se vuelven negativas y relativamente altas para el caso de Colombia y Perú al igual que para el caso de Colombia con Bolivia. Esto se debe a que los efectos globo no son captados de manera explícita en este último modelo sino a través de las correlaciones aparentes entre los términos de error.

Tabla 3. Modelo SUR sin variables de efecto globo

\begin{tabular}{|c|c|c|c|c|c|c|c|c|c|}
\hline \multicolumn{2}{|l|}{ Variables } & Inhccol ${ }_{t}$ & \multicolumn{2}{|c|}{ Inp1usa09 } & & & Ineccol1 $_{(t-1)}$ & dummycol & $\mathrm{R}^{2}$ \\
\hline Ecuación [4] & \multicolumn{2}{|c|}{ endógena } & \multicolumn{2}{|c|}{$\begin{array}{l}-0.342 \\
(0.358)\end{array}$} & & & $\begin{array}{c}0.169 * * * \\
(0.033)\end{array}$ & $\begin{array}{c}-0.394^{* * *} \\
(0.836)\end{array}$ & 0.61 \\
\hline Variables & \multicolumn{2}{|c|}{ Inhcbol $_{t}$} & \multicolumn{2}{|c|}{ Inp1usa09 } & & pibagrbol $_{t}$ & Inecbol1 $_{(\mathrm{t}-1)}$ & dummybol & $\mathrm{R}^{2}$ \\
\hline Ecuación [5] & \multicolumn{2}{|c|}{ endógena } & \multicolumn{2}{|c|}{$\begin{array}{c}0.2315^{\star *} \\
(0.274)\end{array}$} & & $\begin{array}{l}1.19 * * * \\
(3.535)\end{array}$ & $\begin{array}{c}-0.141^{*} \\
(0.088)\end{array}$ & $\begin{array}{c}-0.305^{\star *} \\
(0.143)\end{array}$ & 0.67 \\
\hline Variables & \multicolumn{2}{|c|}{ Inhcper $_{t}$} & \multicolumn{2}{|c|}{ Inp1usa09 } & & & Inecper1 $_{(\mathrm{t}-1)}$ & dummyper & $\mathrm{R}^{2}$ \\
\hline Ecuación [6] & \multicolumn{2}{|c|}{ endógena } & \multicolumn{2}{|c|}{$\begin{array}{c}0.807^{\star *} \\
(0.401)\end{array}$} & & & $\begin{array}{c}-0.1000 \\
(0.108)\end{array}$ & $\begin{array}{l}0.263^{*} \\
(0.159)\end{array}$ & 0.46 \\
\hline \multicolumn{5}{|c|}{ Test de independencia de Breusch-Pagan } & \multicolumn{5}{|c|}{$X^{2}=11.422^{\star \star *}$} \\
\hline \multicolumn{10}{|c|}{ Matriz de correlación de residuales } \\
\hline & Inhccol $_{t}$ & \multicolumn{2}{|c|}{ Inhcbol } & \multicolumn{2}{|c|}{ Inhcper $_{t}$} & & & & \\
\hline Inhccol $\left.\right|_{t}$ & 1.0000 & & & & & & & & \\
\hline Inhcbol & -0.5593 & \multicolumn{2}{|c|}{1.0000} & & & & & & \\
\hline Inhcper $_{t}$ & -0.4880 & \multicolumn{2}{|c|}{0.2288} & \multicolumn{2}{|c|}{1.0000} & & & & \\
\hline
\end{tabular}

*Los errores estándar se encuentran entre paréntesis.

$*<0.1 ; * *<0.05 ; * * *<0.01$

Fuente: cálculos propios con base en las variables construidas. 
Este segundo modelo confirma la fortaleza del modelo SUR y corrobora que hay dos tipos de efectos diferentes que interrelacionan los cultivos ilegales de los tres principales países productores de cocaína:

1) En primer lugar, la presencia de fuertes efectos globo.

2) En segundo lugar, la existencia de complementariedades productivas entre las economías ilegales de los tres países, que constituyen un resultado natural de la integración vertical de toda la cadena y de las ventajas comparativas de cada país en los diferentes eslabones y actividades en ella.

Cabe advertir que las estimaciones realizadas deben leerse mucha con cautela, debido a los problemas de la información sobre las actividades ilegales. En primer lugar, la medición de las variables utilizadas es problemática, ya que algunas de ellas no se captan de forma directa sino a través de estimaciones y aproximaciones estadísticas. Esto se debe a la naturaleza ilegal y criminal de estas actividades. En segundo lugar, las series de que se dispone son cortas. Por todo esto, los modelos estimados no permiten hacer ejercicios de predicción. Se trata de ejercicios de carácter exploratorio que solo permiten obtener evidencia sobre las relaciones de causalidad a nivel cualitativo. Sin embargo, esto último es de gran importancia para darle un mayor sustento analítico -teórico y empírico- al balance y discusión sobre la eficacia que han tenido las políticas de represión de la oferta de drogas en la región andina durante las últimas décadas.

\section{CONCLUSIONES}

Con todo lo anterior, hay evidencia empírica que apoya la presencia de efectos globo en la región andina a lo largo del periodo 1990-2009: el modelo SUR estimado permite corroborar que no existe evidencia estadística suficiente para rechazar la hipótesis de que $a$ lo largo del periodo 1990-2009 se han presentado fuertes efectos globo en el territorio geográfico correspondiente a los principales países productores de cocaína en la región andina: Colombia, Perú y Bolivia. Aunque -como se advirtió- los resultados de los modelos econométricos deben ser leídos con mucha cautela y solo dan indicios del cumplimiento de las hipótesis a nivel cualitativo. Por lo tanto, el modelo muestra que el comportamiento de las hectáreas cultivadas de cualquiera de los tres países productores (Colombia, Perú o Bolivia) depende inversamente de las hectáreas cultivadas en los otros dos países productores.

El modelo también revela otros resultados interesantes: en primer lugar, que los precios de la cocaína al por menor en Estados Unidos inciden de manera proporcional en los cultivos de planta de coca de los tres países productores. Este efecto puede entenderse como un efecto directo o de oferta que tienen 
los precios de los estupefacientes en los lugares de consumo sobre la producción de cocaína y de hoja de coca en los países productores. Esto da indicios de que la baja a largo plazo que se presentó en los precios al por mayor y al por menor en los grandes centros de consumo como Estados Unidos y Europa a lo largo de todo el periodo observado, pudo haber jalonado ceteris paribus una tendencia a la baja en la cantidad de hectáreas cultivadas en los países productores. Sin embargo, este impacto fue contrarrestado - especialmente durante los años noventapor los otros factores que influyeron de manera positiva en el aumento de hectáreas cultivadas, entre ellos la presencia de fuertes efectos globo.

En tercer lugar, como era de esperarse, se halló evidencia de que la proporción del PIB agrícola en Bolivia incide de forma positiva sobre las hectáreas cultivadas con coca en ese país y es altamente significativa. Este resultado se explica por la tradición de consumo de hoja de coca que existe en este país desde épocas remotas

En cuarto lugar, se encontró que la variable de erradicación rezagada $\left(\operatorname{lnecol}_{t-1}\right)$ es significativa para Colombia y tiene signo positivo (contrario a lo esperado). Esto revela que en términos netos -controlando por los demás factores en juego- la erradicación resulta ser contraproducente a lo largo de todo el periodo observado. Esto también corrobora la presencia de efectos hidra (Ortiz, 2002, 2003 y 2009) que implican que la represión puede llevar al resultado paradójico de una expansión de los cultivos ilegales. La incidencia de la variable de erradicación para el caso de Colombia queda más clara al compararla con el signo obtenido para los coeficientes de las variables dummy asociadas al Plan Colombia: aunque la variable dummy con interacción es significativa y tiene un impacto negativo, la dummy de cambio estructural (sin interacción) también es significativa pero está asociada a un coeficiente positivo bastante alto. Esto revela que el Plan Colombia mejoró la eficacia de la represión a las actividades ilegales a través de su interacción con la erradicación. Pero su impacto inicial fue contrarrestado por las fuerzas contrapuestas derivadas de los efectos globo y los efectos hidra.

En quinto lugar, para el caso de Bolivia se encontró que la variable de erradicación rezagada sí tiene un impacto negativo como era de esperarse sobre las hectáreas cultivadas con coca, aunque esta variable solo resultó ser significativa al $10 \%$. En contraste con esto, las variables dummy no resultaron ser significativas para este país. La falta de significancia de las variables dummy probablemente está indicando que en realidad la fuerte represión ejercida a finales de los años noventa a través del llamado "Plan Dignidad", durante el segundo periodo de presidencia de Hugo 
Bánzer, fue inútil; en efecto este punto de vista ha sido defendido por Laserna (2009).

En sexto lugar, para Perú la variable de erradicación no resultó ser significa. Tampoco las variables dummy. Este resultado revela que el impacto neto de la erradicación en ese país controlado por otros factores es irrelevante. Se infiere que el descenso en las hectáreas cultivadas durante los años noventa en ese país no se explica por la represión ejercida por el Gobierno de Fujimori, sino por otros factores: por una parte, la baja de los precios de las drogas duras en Estados Unidos, y por otra, la presencia de fuertes efectos globo.

Por último, el análisis de la estimación alternativa del modelo SUR sin variables de efecto globo indica que hay dos tipos de efectos diferentes que interrelacionan los cultivos ilegales de los tres principales países productores de cocaína: en primer lugar, la presencia de fuertes efectos globo y, en segundo lugar, la existencia de complementariedades productivas entre las economías ilegales de los tres países, que constituyen un resultado natural de la integración vertical de toda la cadena y de las ventajas comparativas de cada país en los diferentes eslabones y actividades en ella.
Las relaciones detectadas y el cumplimiento del efecto globo implican que un plan de represión a la oferta -como el Plan Colombia- aunque útil como una estrategia de guerra contra las drogas focalizada, es inútil como estrategia global cuando no se articula a otros planes rigurosos en los demás países productores. Un plan de represión a la oferta menos inocuo debería diseñarse como una estrategia global, que inhiba la relocalización de los cultivos, de la producción y del tráfico de drogas. Solo de esta manera podría neutralizarse la presencia de efectos globo, tanto en los cultivos ilegales como en el tráfico de los estupefacientes.

Pero, al mismo tiempo, una estrategia global debería exigir que se combata con igual o mayor intensidad el tráfico y la distribución de los estupefacientes en los lugares y países de tránsito hacia los grandes centros de consumo, pero, sobre todo, en estos últimos. Es en los grandes centros de consumo presentes en países como Estados Unidos y en algunos países europeos, donde se obtienen las mayores ganancias de toda la cadena productiva. Por tal razón la interdicción de los alucinógenos y la persecución de los traficantes y distribuidores (al por mayor y al detal) tienen un mayor impacto disuasivo y represivo en las etapas finales de la cadena de valor. 
Los efectos globo en los cultivos de coca en la Región Andina (1990-2009)

Leonardo Raffo López • Javier Andrés Castro • Alexander Díaz España

\section{REFERENCIAS}

Abadie, A., Acevedo, M. C., Kugler, M. \& Vargas J. (2013). Inside the War on Drugs: Effectiveness and Unintended Consequences of a Large Illicit Crops Eradication Program in Colombia. Working Paper Harvard Kennedy School and Universidad del Rosario.

Arce, M. \& Reales, L. (2006). Violencia política, asistencia militar de estados unidos y producción de coca en los andes centrales. Revista de Ciencia Política, 26(1), 25 47.

Bagley, B. (1988). Colombia and the War on Drugs. Foreign Affairs, 67(1),70-92.

Bagley, B. (2012). Drug Trafficking and Organized Crime in the Americas. Woodrow Wilson Center Update of the Americas. Retrieved from https:/www.wilsoncenter. org/sites/default/files/BB\%20Final.pdf

Becker, G. S., Murphy K. \& Grossman, M. (2006). El mercado de bienes ilegales: el caso de la droga. Revista de Economía Institucional, 8(15), 17-42.

Becker, G. S., Murphy K. \& Grossman, M. (2006). The Market for Illegal Goods: The Case of Drugs", Journal of Political Economy, 114(1), 38-60.

Botero, H. (2013). The Effects of Drug Enforcement on Violence in Colombia 1999-2010: A Spatial Econometric Approach, Munich Personal RePEc Archive, (49459). Recuperado de http://mpra.ub.uni-muenchen.de/49459/1/MPRA_paper_49459.pdf.

Caicedo, C. J. (2006). Expectativas racionales y persistencia de los cultivos ilícitos de coca en Colombia 1986-2003. Bogotá, Colombia: CIDER, Universidad de los Andes.

Caulkins, J.P., Crawford G. \& Reuter P. (1993). Simulation of Adaptive Response: A Model of Interdictor-Smuggler Interactions. Mathematical and Computer Modelling, 17(2), 37-52.

Dávalos, L. M., Bejarano, A. C., \& Correa, H. L. (2009). Disabusing Cocaine: Pervasive Myths and Enduring Realities of a Globalised Commodity. International Journal of Drug Policy, 20(5), 381-386.

Dávalos, L. M., Bejarano, A. C., Hall, M. A., Correa, H. L., Corthals, A. \& Espejo, O. J. (2011). Forests and Drugs: Coca-driven Deforestation in Tropical Biodiversity Hotspots. Environmental science \& technology, 45(4), 1219-1227.

Dion, M. L. \& Russler, C. (2008). Eradication Efforts, the State, Displacement and Poverty: Explaining Coca Cultivation in Colombia during Plan Colombia. Journal of Latin American Studies, 40(3), 399-421. 
Díaz, A. M. \& Torres, F. (2004). Geografía de los cultivos ilícitos y conflicto armado en Colombia. Documento CEDE, (18).

Duncan, G. (2014). Más que plata o plomo: el poder político del narcotráfico en Colombia y México, (1ed.). Bogotá: Debate.

Greene, W. (2000). Econometric Analysis. (5 ed.). New Jersey: Prentice Hall.

Laserna, R. (2009). La política de drogas en Bolivia. En J.G. Tokatlian (Ed.) La guerra contra las drogas en el mundo andino (pp.17-49). Buenos Aires: Libros de Zorzal.

Mejía, D. \& Restrepo, P. (2008). The War on Illegal Drug Production and Trafficking: an Economic Evaluation of Plan Colombia, Documento CEDE, (19).

Mejía D. \& Restrepo, P. (2011). Políticas antidroga en el Plan Colombia: costos, efectividad y eficiencia. En A. Gaviria A. \& D. Mejía (eds.) Políticas antidroga en Colombia: éxitos, fracasos y extravíos (pp. 413-445). Bogotá: Universidad de los Andes.

Mejía, D. \& Restrepo, P. (2013). The Economics of the War on Illegal Drug Production and Trafficking, Working Paper, Stanford. Retrieved from http://crimelab.stanford.edu/clftp/uploads/183/5838.pdf.

Moreno-Sánchez, R., Kraybill, D. S. \& Thompson, S. R. (2003). An Econometric Analysis of Coca Eradication Policy in Colombia. World Development, 31(2), 375-383.

Nadelmann E. (1989). Drug Prohibition in America: Costs, Consequences and Alternatives. Science (253), 949-957.

Ortiz, C. H. (2002). Luchando infructuosamente contra la hidra: un modelo sencillo del narcotráfico, Cuadernos de Economía, 37.

Ortiz, C. H. (2003). La guerra contra las drogas es contraproducente: un análisis económico de equilibrio general. Lecturas de Economía, 58, 47-68.

Ortiz, C. H. (2009). The War on Drugs is Counterproductive, Once Again. Revista Lecturas de Economía, 71, 19-42.

Posada, C. E. \& Mejía D. (2007). Cocaine Production and Trafficking What do we Know? World Bank Publications.

Raffo, L. \& Segura, J.L. (2015). Las redes del narcotráfico y sus interacciones: un modelo teórico. Revista de Economía Institucional 17(32), 183-212.

Reuter, P. (2014). The Mobility of Drug Trafficking. In LSE Expert Group on the Economics of Drug Policy (Ed.), Ending the Drug Wars (pp.33-40). London: LSE. 
Reyes, L. C. (2014). Estimating the causal effect of forced eradication on coca cultivation in Colombian municipalities. World Development, 61, 70-84.

Rincón-Ruiz, A. \& Kallis, G. (2013). Caught in the Middle, Colombia's War on Grugs and its Effects on Forest and People. Geoforum, 46, 60-78.

Rojas, D. M. (2007). Plan Colombia II: ¿más de lo mismo? Revista Colombia Internacional, (65), 14-37.

Rouse, S. \& Arce, M. (2006). The Drug-Laden Balloon: US Military Assistance and Coca Production in the Central Andes. Social Science Quarterly, 87(3), 540-557.

Rozo, S. V. (2012). On the Effectiveness and Welfare Consequences of Anti-drug Eradication Programs. Working Paper, Bogotá: Universidad de los Andes.

Soberón, R. (2009). Hacia una nueva perspectiva de la temática del tráfico ilícito de estupefacientes: el caso del Perú. En J.G. Tokatlian (Ed.), La guerra contra las drogas en el mundo Andino (pp.165-212). Buenos Aires: Libros de Zorzal.

Thoumi, F. (2009). Las drogas ilegales, el fracaso de la política antinarcóticos y la necesidad de reformas institucionales en Colombia. En J.G Tokatlian (Ed.), La guerra contra las drogas en el mundo andino (pp.51-123). Buenos Aires: Libros de Zorzal.

UNODC (1999, 2000, 2006, 2008, 2009. 2010, 2011, 2012 y 2013). World Drug Report. New York: UN. 